\title{
Heart rate variability in athletes and nonathletes at rest and during head-up tilt
}

F.S. Martinelli, ${ }^{1,3}$,

M.P.T. Chacon-Mikahil2,

L.E.B. Martins ${ }^{1}$,

E.C. Lima-Filho ${ }^{1 \dagger}$,

R. Golfetti ${ }^{1}$, M.A. Paschoal ${ }^{1,5}$ and L. Gallo-Junior ${ }^{4}$
Correspondence

F.S. Martinelli

Laboratório de Fisiologia do

Exercício

Faculdade de Educação Física UNICAMP

Av. Érico Veríssimo, 701

13083-970 Campinas, SP

Brasil

E-mail: chezfab@terra.com.br

Research supported by FAPESP (No. 96/09945-0) and CNPq

(No. 300528/85-0).

Received July 14, 2003

Accepted January 24, 2005
${ }^{1}$ Laboratório de Fisiologia do Exercício, ²Departamento de Ciências do Esporte, Faculdade de Educação Física, ${ }^{3}$ Departamento de Fisiologia e Biofísica, Instituto de Biologia, Universidade Estadual de Campinas, Campinas, SP, Brasil

${ }^{4}$ Divisão de Cardiologia, Departamento de Clínica Médica, Hospital das Clínicas, Faculdade de Medicina de Ribeirão Preto, Universidade de São Paulo, Ribeirão Preto, SP, Brasil

${ }^{5}$ Departamento de Fisioterapia Cardiorespiratória, Faculdade de Fisioterapia, Pontifícia Universidade Católica de Campinas, Campinas, SP, Brasil

\begin{abstract}
The purpose of the present study was to determine if autonomic heart rate modulation, indicated by heart rate variability (HRV), differs during supine rest and head-up tilt (HUT) when sedentary and endurance-trained cyclists are compared. Eleven sedentary young men (S) and 10 trained cyclists $(\mathrm{C})$ were studied. The volunteers were submitted to a dynamic ECG Holter to calculate $\mathrm{HRV}$ at rest and during a $70^{\circ}$ HUT. The major aerobic capacity of athletes was expressed by higher values of $\dot{\mathrm{VO}}_{2}$ at anaerobic threshold and peak conditions $(\mathrm{P}<0.05)$. At rest the athletes had lower heart rates $(\mathrm{P}<0.05)$ and higher values in the time domain of HRV compared with controls (SD of normal RR interval, SDNN, medians): $59.1 \mathrm{~ms}$ (S) vs $89.9 \mathrm{~ms}$ (C), $\mathrm{P}<0.05$. During tilt athletes also had higher values in the time domain of HRV compared with controls (SDNN, medians): $55.7 \mathrm{~ms}$ (S) vs $69.7 \mathrm{~ms}$ (C), $\mathrm{P}<0.05$. No differences in power spectral components of HRV at rest or during HUT were detected between groups. Based on the analysis of data by the frequency domain method, we conclude that in athletes the resting bradycardia seems to be much more related to changes in intrinsic mechanisms than to modifications in autonomic control. Also, HUT caused comparable changes in sympathetic and parasympathetic modulation of the sinus node in both groups.
\end{abstract}

\section{Introduction}

Several studies have reported that aerobic physical training changes the sympathovagal balance of the sinus node and may contribute in part to the resting bradycardia observed in endurance athletes. However, it
Key words

- Endurance

- Power spectral analysis

- Heart rate variability

- Autonomic heart rate modulation

- Training bradycardia

- Orthostatic stress 
alterations are responsible for endurancetrained bradycardia (7-12).

In addition to the possible role of vagal and sympathetic adaptations in resting bradycardia induced by aerobic training, different autonomic cardiovascular responses may be activated during exercise itself and in postural and respiratory tests when trained and untrained subjects are compared $(11,13)$. Some studies have reported that during ortosthatic stress, trained and untrained subjects present different cardiovascular responses, which have been associated with alterations in autonomic cardiovascular control $(5,14,15)$. However, other studies failed to show these differences when comparing trained and untrained subjects $(16,17)$.

In order to evaluate the cardiac autonomic activity non-invasively, many studies have used the measurement of the heart rate variability (HRV) that is obtained by calculating the variations between the RR intervals of the ECG signal. These variations are calculated in the time and frequency domains; in this last condition they are expressed as sine wave power values (power spectrum). High HRV is usually caused by an increase in vagal tone associated with a decrease in sympathetic tone $(18,19)$.

There is substantial controversy concerning the absolute and relative changes of power spectral components related to sympathetic and parasympathetic modulation, induced by aerobic training $(1,4,6,14,20-23)$.

Thus, the aim of this study was to evaluate $\mathrm{HRV}$ in the time and frequency domains in athletes and sedentary subjects in the supine position and after head-up tilt (HUT), when there is a change in sympathetic and parasympathetic balance in the sinus node.

\section{Material and Methods}

\section{Subjects}

Two groups of young men, nonsmoking healthy volunteers, were studied: 11 seden- tary controls, who had not engaged in regular physical activity for at least 6 months, and 10 trained cyclists of competitive level, who had been participating in an endurance training program for at least one year. They trained $90 \mathrm{~min}$ to $5 \mathrm{~h} / \mathrm{session}, 6$ days a week and all participated in official national and/ or international championships in this athletic modality. Their anthropometric characteristics are presented in Table 1.

The study was approved by the Ethics Committee of the State University of Campinas, Campinas, SP, Brazil, and each subject was informed about the nature of the experiment and gave their informed written consent.

\section{Protocols}

The protocols were conducted in two separate sessions. In the first all subjects performed a dynamic physical exercise test on a cycloergometer (Quinton Instrument Company, Seattle, WA, USA), using a progressive continuous protocol (ramp) up to physical exhaustion. The initial workload was 4 watts for 2 min for both groups. The workload increases were 15 $\mathrm{W}$ per min for the sedentary group and $30 \mathrm{~W}$ per min for the trained group. The subjects pedaled at a frequency of $60 \mathrm{rpm}$. The ventilatory and metabolic variables were obtained by direct measurement using a gas analyzer system (metabolic measuring chart-horizon systems; Sensormedics Corporation, Yorba Linda, CA, USA). The ECG was recorded continuously starting one minute before the beginning of the exercise and up to the end of the recovery period. The ventilatory anaerobic threshold was obtained by analyzing the loss of linearity of $\mathrm{CO}_{2}$ production and pulmonary ventilation responses at submaximal power values (as averaged at 15-s intervals) (24).

In the second session, which was held at least $24 \mathrm{~h}$ after the exercise test, all subjects initially rested for $40 \mathrm{~min}$ in the supine position with continuous recording of heart rate obtained by automatic counting of the $\mathrm{R}$ wave 
peaks of an ECG signal. After the supine resting period, a passive $70^{\circ}$ HUT was performed without foot support using a seat attached to the table to support body weight. The subjects were tilted and remained in this position for up to $60 \mathrm{~min}$.

The ECG, the instantaneous heart rate, the pulse pressure, and the respiratory rhythm were recorded continuously with an eightchannel recording system (RS 3800; Gould Instruments Systems, Valley View, OH, USA).

Tests were performed in a quiet air-conditioned $\left(22-24^{\circ} \mathrm{C}\right)$ room. The subjects were instructed to perform no exercise training $40 \mathrm{~h}$ before the day of the experiment and to avoid drugs and caffeine $12 \mathrm{~h}$ before the test.

\section{Heart rate variability}

The short-term HRV in the time and frequency domains was obtained using 24-h dynamic electrocardiography (Holter for Windows, v. 3.6-F, Rozinn Electronics, Glendale, NY, USA). Two or more 5-min windows that presented stationarity (visual inspection) were selected from the RR interval series during rest and HUT.

During HUT, HRV was analyzed after the initial transient response of this variable, always during periods of stationarity of the signal. Since autonomic heart rate control cannot be the same at the beginning and at the end of HUT, linear regression analysis was performed to determine if the time of tilting was correlated with the HRV responses. This analysis showed that all 5-min stationary windows during this maneuver could be used. Then, each subject had a different number (average $=3$ ) of 5-min intervals for calculating the HRV, with the first interval being analyzed at least $6 \mathrm{~min}$ after tilting. When these intervals were consecutive for the subject, their arithmetic mean was used. The non-consecutive intervals were considered separately as repetitions.

The following HRV variables were ana- lyzed: for the time domain - standard deviation of normal RR interval (SDNN, ms); for the frequency domain - low frequency power in normalized units (LFnu, 0.04-0.15 Hz), high frequency power in normalized units (HFnu, 0.15-0.4 Hz), and LF/HF - ratio of absolute LF power to HF power.

The spectral analysis was calculated by the MK5 Spectral Analysis Package, H4W Rozinn Electronics, using the Fast Fourier Transform algorithm. The sampling rate was 128 samples per second and all data were analyzed in blocks of $100 \mathrm{~s}$, with a frequency of resolution of $0.01 \mathrm{~Hz}$. The spectra of six successive 100 -s blocks were averaged together.

Visual inspection of ECG signal recordings in the computer monitor showed absence of artifacts or cardiac arrhythmias that could interfere with the HRV analysis performed by the system analyzer (Holter for Windows, v.3-6-F Rozinn Electronics).

\section{Statistical analysis}

Since most of the data did not present Gaussian distributions, nonparametric tests were used for statistical analysis. The statistical procedure used is the one proposed in McGill et al. (25) for exploratory data analysis for various groups. Differences between groups were demonstrated by the confidence interval of the median presented in the boxplots, with the level of significance set at alpha $=0.05$. If the intervals in the two boxes do not overlap, this indicates a difference in a location at roughly the $5 \%$ level. The same procedure is used for paired sample observations when the differences of the pairs are used as a single sample of observations (26). The exact distribution for the statistical test used to compute the probability value whenever possible, in case of no tie samples, is the Wilcoxon rank sum test for two sample data (equivalent to the Mann-Whitney test) or the Wilcoxon signed rank test for paired or one sample data. 
Table 1. Anthropometric and cardiorespiratory parameters of the sedentary and endurance-trained subjects at rest and during exercise.

\begin{tabular}{lcc}
\hline & Sedentary group $(\mathrm{N}=11)$ & Cyclists $(\mathrm{N}=10)$ \\
\hline Age (years) & $21.82(2.18)$ & $20.80(3.33)$ \\
Height $(\mathrm{cm})$ & $175.36(6.70)$ & $175.55(5.82)$ \\
Weight $(\mathrm{kg})$ & $67.14(8.56)$ & $70.59(9.61)$ \\
Body surface area $\left(\mathrm{m}^{2}\right)$ & $1.80(0.14)$ & $1.84(0.15)$ \\
HR & $70.9(10.75)$ & $65.6(10.49)^{*}$ \\
$\mathrm{HR}_{\text {AT }}(\mathrm{bpm})$ & $130.45(15.41)$ & $146.7(15)^{*}$ \\
$\mathrm{HR}$ peak $(\mathrm{bpm})$ & $195.73(12.04)$ & $189.7(9.12)$ \\
$\mathrm{P}_{\text {AT }}(\mathrm{W})$ & $108.48(38.36)$ & $209.25(30.65)^{*}$ \\
$\mathrm{P}_{\text {peak }}(\mathrm{W})$ & $212.27(29.12)$ & $358.8(19.68)^{*}$ \\
$\dot{V O}_{2 A T}\left(\mathrm{ml} \mathrm{kg} \mathrm{kg}^{-1} \mathrm{~min}^{-1}\right)$ & $19.55(6.53)$ & $35.16(6.26)^{*}$ \\
$\dot{V O}_{2 \text { peak }}\left(\mathrm{ml} \mathrm{kg}^{-1} \mathrm{~min}^{-1}\right)$ & $38.97(6.79)$ & $57.01(4.82)^{*}$ \\
\hline
\end{tabular}

Data are reported as means $(\mathrm{SD})$. $\mathrm{HR}_{\text {rest }}=$ heart rate at supine rest; $\mathrm{HR}_{\mathrm{AT}}=$ heart rate at anaerobic threshold; $\mathrm{HR}_{\text {peak }}=$ heart rate at peak effort; $\mathrm{P}_{\mathrm{AT}}=$ power at anaerobic threshold; $\mathrm{P}_{\text {peak }}=$ power at peak effort; $\dot{\mathrm{V}} \mathrm{O}_{2 \mathrm{AT}}=$ oxygen uptake at anaerobic threshold; $\dot{\mathrm{V}} \mathrm{O}_{2 \text { peak }}=$ oxygen uptake at peak effort.

${ }^{*} \mathrm{P}<0.05$ compared to sedentary group (Wilcoxon rank sum test).

Table 2. Comparison of heart rate variability parameters in time and frequency domains in the supine and tilting position.

\begin{tabular}{|c|c|c|c|c|}
\hline & \multicolumn{2}{|c|}{ Supine position } & \multicolumn{2}{|c|}{ Tilting position } \\
\hline & $\begin{array}{l}\text { Sedentary group } \\
\qquad(N=11)\end{array}$ & $\begin{array}{l}\text { Cyclists } \\
(N=10)\end{array}$ & $\begin{array}{l}\text { Sedentary group } \\
\qquad(N=11)\end{array}$ & $\begin{array}{l}\text { Cyclists } \\
(N=10)\end{array}$ \\
\hline $\mathrm{RR}$ mean (ms) & $854.3(105.7)$ & $1076(115.6)^{*}$ & $625.8(188.5)^{+}$ & $794.2(148)^{*+}$ \\
\hline SDNN (ms) & $59.1(36.5)$ & $89.9(24.8)^{*}$ & 55.7 (8.8) & $69.7(18.9)^{*+}$ \\
\hline $\mathrm{LF}\left(\mathrm{ms}^{2} / \mathrm{Hz}\right)$ & $3500(2900)$ & 8400 (3300) & $4700(1050)$ & 8000 (7400) \\
\hline $\mathrm{HF}\left(\mathrm{ms}^{2} / \mathrm{Hz}\right)$ & $1500(800)$ & $2400(900)$ & $770(550)$ & 880 (975) \\
\hline LFnu (\%) & $71(6)$ & $68(20)$ & $85(4.5)^{+}$ & $88(8)^{+}$ \\
\hline HFnu (\%) & $29(6)$ & $33(18)$ & $15(4.8)^{+}$ & $12(8.2)^{+}$ \\
\hline $\mathrm{LF} / \mathrm{HF}$ & $2.47(1.01)$ & $2.08(1.29)$ & $5.56(2.48)^{+}$ & $7.24(6.83)^{+}$ \\
\hline
\end{tabular}

Data are reported as medians and interquartile range. SDNN = standard deviation of normal RR interval; LF, HF = low and high frequency power, respectively; LFnu = LF power in normalized units; HFnu = HF power in normalized units; LF/HF = ratio of absolute LF power to HF power values. Frequency ranges: LF: $0.04-0.15 \mathrm{~Hz}$ and HF: $0.15-0.4 \mathrm{~Hz}$.

${ }^{*} \mathrm{P}<0.05$ compared to the sedentary group; ${ }^{+P}<0.05$ between the supine and tilting position (Wilcoxon rank sum test and Wilcoxon signed rank test, respectively).

Figure 1. Mean RR in the supine and head-up tilt (HUT) position for the sedentary group and cyclists. The boxplots show the minimum values, 1st quartile, medians (bold black line), 3rd quartile, maximum, and confidence interval of the medians (hatched area). $\mathrm{S}=$ sedentary group $(N=11) ; C=$ cyclists $(N=10)$. ${ }^{*} P<0.05$ for comparisons indicated by brackets (confidence interval of the median test).

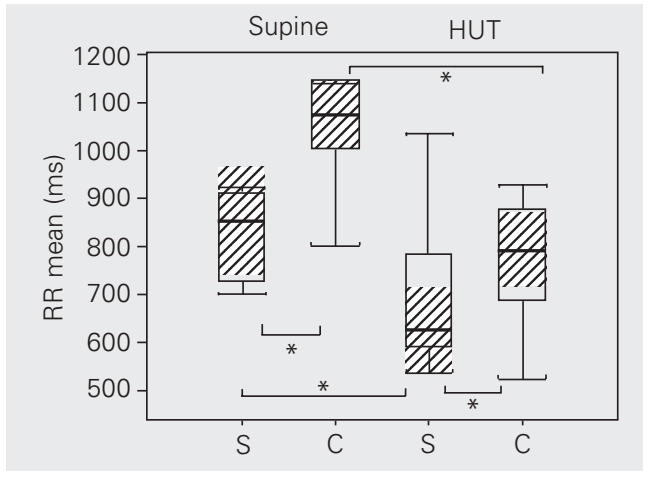

\section{Results}

\section{Cardiorespiratory variables at rest and during exercise}

Table 1 shows the higher aerobic capacity of the athletes compared to control group, expressed by higher $(\mathrm{P}<0.05)$ oxygen uptake and power values at anaerobic threshold $\left(\dot{\mathrm{VO}}_{2 \mathrm{AT}}\right)$ and at peak condition $\left(\dot{\mathrm{VO}}_{2 \text { peak }}\right)$. The heart rate in the resting condition was significantly $(\mathrm{P}<0.05)$ lower in athletes than in nonathletes (Table 1).

\section{Heart rate variability at rest}

Table 2 and Figure 1 show that the RR mean (medians) in the supine position was higher $(\mathrm{P}<0.05)$ in athletes than in nonathletes. Figures 2 to 5 show that, despite the significantly $(\mathrm{P}<0.05)$ lower values of SDNN in the sedentary group, the spectral power components, LFnu, HFnu and LF/HF, were similar for the two groups.

\section{Heart rate variability during head-up tilt}

During HUT (Table 2 and Figures 1 to 5) both groups presented reduction in $\mathrm{RR}$ mean values $(\mathrm{P}<0.05)$. Athletes also presented reductions in SDNN $(\mathrm{P}<0.05)$. In both groups, spectral components showed the following results: higher LFnu and $\mathrm{LF} / \mathrm{HF}$ ratio $(\mathrm{P}<0.05)$ and lower HFnu $(\mathrm{P}<0.05)$. Higher values $(\mathrm{P}<0.05)$ of mean $\mathrm{RR}$ and SDNN were found in athletes, but no differences in power spectral variables were found between groups.

\section{Discussion}

The higher aerobic capacity of the athletes was reflected in greater $\mathrm{VO}_{2}$ and power values (cycloergometer) at both anaerobic threshold and peak effort (Table 1). Also, the resting heart rate was significantly lower in cyclists than in nonathletes. Several inves- 

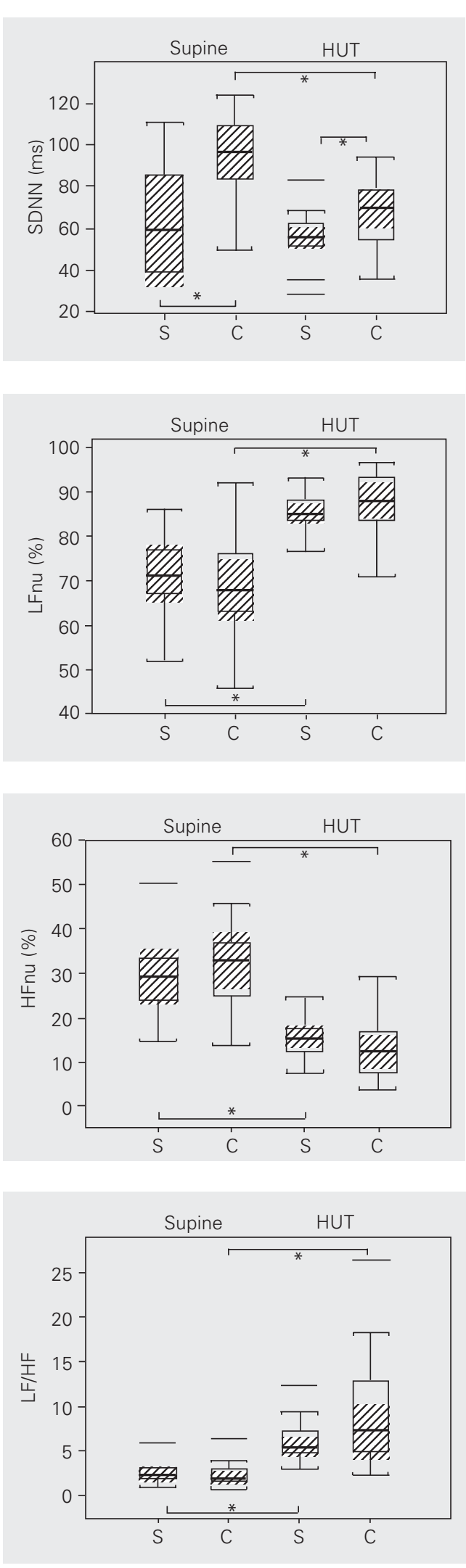

Figure 2. Standard deviation of normal RR interval in the supine and head-up tilt position for the sedentary subjects and cyclists. The boxplots show the minimum values, 1st quartile, medians (bold black line), 3rd quartile, maximum, and confidence interval of the medians (hatched area). $S=$ sedentary group $(N=11) ; C=$ cyclists $(N=10)$; HUT = head-up tilt; SDNN = standard deviation of normal $R R$ interval. ${ }^{*} P<0.05$ for comparisons indicated by brackets (confidence interval of the median test and Wilcoxon rank sum test).

Figure 3. Low frequency in normalized units in the supine and head-up tilt position for the sedentary subjects and cyclists. The boxplots show the minimum values, 1st quartile, medians (bold black line), 3rd quartile, maximum, and confidence interval of the medians (hatched area). $\mathrm{S}=$ sedentary group $(\mathrm{N}=11) ; \mathrm{C}=$ cyclists $(\mathrm{N}=$ 10); HUT = head-up tilt; LFnu = low frequency in normalized units. ${ }^{*} P<0.05$ for comparisons indicated by brackets (confidence interval of the median test).

Figure 4. High frequency in normalized units at supine and tilting position in the sedentary subjects and cyclists. The boxplots show the minimum values, 1st quartile, medians (bold black line), 3rd quartile, maximum, and confidence interval of the medians (hatched area). $S=$ sedentary group $(N=11) ; C=$ cyclists $(N=$ 10); HUT = head-up tilt; HFnu = high frequency in normalized units. ${ }^{*} \mathrm{P}<0.05$ for comparions indicated by brackets (confidence interval of the median test).

Figure 5. Low frequency-high frequency ratio (absolute values) in the supine and head-up tilt position for the sedentary subjects and cyclists. The boxplots show the minimum values, 1 st quartile, medians (bold black line), 3rd quartile, maximum, and confidence interval of the medians (hatched area). $\mathrm{S}=$ sedentary group $(\mathrm{N}=$ 11); $C=$ cyclists $(N=10) ; H U T=$ head-up tilt; $L H / H F=$ low frequency-high frequency ratio. ${ }^{*} P<0.05$ for comparisons indicated by brackets (confidence interval of the median test). 
tigations have been conducted regarding the mechanisms responsible for the resting bradycardia in trained subjects. Changes in the intrinsic mechanisms acting on the sinus node and alterations in the autonomic nervous system control of the heart have been reported to contribute to this phenomenon. In this context, some studies using different tests like pharmacological blockade, respiratory sinus arrhythmia and HRV have reported that enhanced vagal tone and/or diminished sympathetic activity might contribute in part to the resting bradycardia (1$4,6)$. In contrast, studies conducted on animals and men using the same methodology have related the training resting bradycardia to reduced intrinsic HR (8-12,27).

Several cross-sectional and longitudinal studies have compared resting HRV between endurance-trained and untrained subjects. Some have shown that endurance-trained subjects present higher values of power spectral HRV, indicating increasing vagal activity $(1,3,4,6,28)$; others have reported higher values of the LF component of HRV suggesting higher sympathetic activity $(4,14,29)$. Lower levels of sympathetic activation (1) and vagal activation (29) and, finally, no differences have been found between trained and untrained subjects (10). The results regarding time domain HRV are also controversial, and some studies have shown differences in time domain that were not accompanied by corresponding differences in power spectral HRV (23). Migliaro et al. (30) failed to show a relationship between fitness and $\mathrm{HRV}$ in the time and frequency domains and supported the idea that age and heart rate are the main determinants of HRV.

In the present study, time domain analysis of HRV in the supine position showed higher values in athletes, but it is important to note that the standard deviation by itself (SDNN) does not permit the determination of which division of the autonomic system is responsible for the change (7). However, in the power spectral analysis, the HFnu com- ponent of HRV, which has been associated with vagal efferent activity $(7,31,32)$, did not differ between athletes and nonathletes. The LFnu component also did not differ between groups. However, this component may not express the separate modulation of the sympathetic system; the parasympathetic system may contribute to the LF component in physiological conditions (7,31). Finally, the LF/ HF ratio, which indicates the sympatho-vagal balance (32), also did not differ between athletes and nonathletes.

These results show that endurance-trained athletes present indications of increased HRV in the time domain but not in the frequency domain. To explain these findings one must consider the following possibilities. First, mean RR values were very different between athletes and nonathletes and it was previously shown that time domain variables are strongly influenced by mean RR values $(4,10)$. Second, in the case of athletes, if the RR interval length is close to the maximally attainable value, the corresponding changes in RR cycle length can only change in one direction, potentially limiting the amplitude of the power spectrum (23). Third, the cumulative effect of heavy training (cyclism training) performed by the athletes may have contributed to higher LFnu and lower HFnu values in this group (14, $22,29)$. Fourth, some investigators have proposed that frequency domain methods are more indicated than time domain methods for short-term assessment of HRV, as was the case in the present study (7). Finally, an alternative explanation for the lack of differences in the power spectral components between athletes and nonathletes could be the inaccuracy of power spectral analysis to assess autonomic tone in a highly trained population (23), or even the occurrence of nonlinearity components in autonomic control systems.

Despite these possible methodological problems, the absence of differences in the spectral variables between athletes and 
nonathletes suggests that a non-autonomic mechanism plays a major role in the resting bradycardia of cyclists. In agreement with this hypothesis, Yamamoto et al. (28), in a longitudinal study, detected cardiac autonomic adaptation up to the 28th day of endurance training, with no further changes being detected thereafter despite a continued decrease in heart rate. Bonaduce et al. (33) also observed that decreased intrinsic heart rate plays an important role in the resting bradycardia induced by endurance training. They reported that detrained athletes showed a higher cardiac parasympathetic modulation with lower heart rate than controls, but after vigorous training no further increase in parasympathetic modulation was detectable despite further decreases in heart rate.

The present study also investigated if differences in autonomic cardiovascular control could appear between athletes and nonathletes during a situation of orthostatic stress, which is a natural stimulus leading to sympathetic excitation and vagal withdrawal in the heart. During HUT the initial increase in the heart rate response is mediated by a decrease in vagal activity, followed by an enhanced sympathetic activity that maintains higher values of heart rate during the test (34). Our results agree with previous observations showing an increase in heart rate (reduced RR mean) and enhanced sympathetic activity (expressed by higher LFnu values) associated with a decreased vagal activity (expressed by lower HFnu values) during HUT in both groups.

There are few HRV studies concerning the responses of sedentary and endurancetrained athletes to maneuvers that reduce blood return to the heart, such as active standing, passive HUT and lower body negative pressure. Dixon et al. (1) compared sed- entary subjects and long distance runners during active standing and did not find differences in power spectral HRV between them. However, Furlan et al. (14) compared sedentary subjects and endurance-trained athletes during tilt and found indications of higher sympathetic activity in athletes, expressed by higher values of LF and LF/HF ratio. When comparing athletes before and after a training period of 7 months, Hedelin et al. (35) showed that improved measures of aerobic capacity were associated with reduction of LF HRV in the tilted position, while HF and total HRV did not change. They also suggested that high levels of HF and total HRV seem to constitute a favorable condition for an increase in $\mathrm{VO}_{2}$ max rather than being a result of increased aerobic fitness. Most recently, Lee (36) showed that 8 endurance training sessions reduced LF and LF/HF during 5-min tilt and paced breathing, but not during spontaneous breathing. In the present study, vagal and sympathetic activity during HUT performed with spontaneous breathing did not seem to differ between the cyclists and sedentary group, considering the power spectral components. SDNN was higher in athletes than in sedentary subjects during HUT, but the same methodological limitations mentioned above have to be considered.

The results of the present cross-sectional study suggests that the aerobic physical training adaptations observed in cyclists were not associated with alterations in autonomic cardiovascular responses at rest or during HUT, as evaluated by frequency domain HRV. Furthermore, our data indicate that the bradycardia found in endurance-trained athletes appears to be related to intrinsic adaptations in the sinus node. 


\section{References}

1. Dixon EM, Kamath MV, McCartney N \& Fallen EL (1992). Neural regulation of heart rate variability in endurance athletes and sedentary controls. Cardiovascular Research, 26: 713-719.

2. Ekblom B, Kilbom A \& Soltysiak J (1973). Physical training, bradycardia, and autonomic nervous system. Scandinavian Journal of Clinical and Laboratory Investigation, 32: 251-256.

3. Goldsmith RL, Bigger Jr JT, Steinman RC \& Fleiss JL (1992). Comparison of 24-hour parasympathetic activity in endurance-trained and untrained young men. Journal of the American College of Cardiology, 20: 552-558.

4. Puig J, Freitas J, Carvalho MJ, Puga N, Ramos J, Fernandes P, Costa O \& de Freitas AF (1993). Spectral analysis of heart rate variability in athletes. Journal of Sports Medicine and Physical Fitness, 33: 44-48.

5. Shi X, Stevens GH, Foresman BH, Stern SA \& Raven PB (1995). Autonomic nervous system control of the heart: endurance exercise training. Medicine and Science in Sports and Exercise, 27: 1406-1413.

6. Shin K, Minamitani H, Onishi S, Yamazaki H \& Lee M (1997). Autonomic differences between athletes and nonathletes: spectral analysis approach. Medicine and Science in Sports and Exercise, 29: 1482-1490.

7. Hartikainen JEK, Tahvannainen KUO \& Kuusela TA (1998). Shortterm measurement of heart rate variability. In: Malik M (Editor), Clinical Guide to Cardiac Autonomic Tests. Kluwer Academic Publishers, Norwell, MA, USA.

8. Hatfield BD, Spalding TW, Santa Maria DL, Porges SW, Potts JT, Byrne EA, Brody EB \& Mahon AD (1998). Respiratory sinus arrhythmia during exercise in aerobically trained and untrained men. Medicine and Science in Sports and Exercise, 30: 206-214.

9. Katona PG, McLean M, Dighton DH \& Guz A (1982). Sympathetic and parasympathetic cardiac control in athletes and nonathletes at rest. Journal of Applied Physiology, 52: 1652-1657.

10. Loimaala A, Huikuri H, Oja P, Pasanen M \& Vuori I (2000). Controlled 5-mo aerobic training improves heart rate but not heart rate variability or baroreflex sensitivity. Journal of Applied Physiology, 89: 18251829.

11. Maciel BC, Gallo Junior L, Marin Neto JA, Lima Filho EC, Terra Filho J \& Manco JC (1985). Parasympathetic contribution to bradycardia induced by endurance training in man. Cardiovascular Research, 19: 642-648

12. Stein RMC, Rosito GA, Zimerman LI \& Ribeiro J (2002). Intrinsic sinus and atrioventricular node electrophysiologic adaptations in endurance athletes. Journal of the American College of Cardiology, 39: 1033-1038.

13. Gallo Jr L, Maciel BC, Marin-Neto JA, Martins LE, Lima-Filho EC, Golfetti R, Chacon MP \& Forti VA (1995). Control of heart rate during exercise in health and disease. Brazilian Journal of Medical and Biological Research, 28: 1179-1184.

14. Furlan R, Piazza S, Dell'Orto S, Gentile E, Cerutti S, Pagani M \& Malliani A (1993). Early and late effects of exercise and athletic training on neural mechanisms controlling heart rate. Cardiovascular Research, 27: 482-488.

15. Zhang LF, Zheng J, Wang SY, Zhang ZY \& Liu C (1999). Effect of aerobic training on orthostatic tolerance, circulatory response, and heart rate dynamics. Aviation, Space, and Environmental Medicine, 70: 975-982.

16. Lansimies EA \& Rauhala E (1986). Orthostatic tolerance and aerobic capacity. Aviation, Space, and Environmental Medicine, 57 (Part 1): 1158-1164.

17. Lightfoot JT, Claytor RP, Torok DJ, Journell TW \& Fortney SM (1989). Ten weeks of aerobic training do not affect lower body negative pressure responses. Journal of Applied Physiology, 67: 894-901.

18. Akselrod S, Gordon D, Ubel FA, Shannon DC, Berger AC \& Cohen RJ (1981). Power spectrum analysis of heart rate fluctuation: a quantitative probe of beat-to-beat cardiovascular control. Science, 213: $220-222$.

19. Anonymous (1996). Heart rate variability: standards of measurement, physiological interpretation and clinical use. Task Force of the European Society of Cardiology and the North American Society of Pacing and Electrophysiology. Circulation, 93: 1043-1065.

20. De Meersman RE (1993). Heart rate variability and aerobic fitness. American Heart Journal, 125: 726-731.

21. Goldsmith RL, Bigger Jr JT, Bloomfield DM \& Steinman RC (1997). Physical fitness as a determinant of vagal modulation. Medicine and Science in Sports and Exercise, 29: 812-817.

22. Melanson EL (2000). Resting heart rate variability in men varying in habitual physical activity. Medicine and Science in Sports and Exercise, 32: 1894-1901.

23. Sacknoff DM, Gleim GW, Stachenfeld N \& Coplan NL (1994). Effect of athletic training on heart rate variability. American Heart Journal, 127: $1275-1278$.

24. Wasserman K, Whipp BJ, Koyl SN \& Beaver WL (1973). Anaerobic threshold and respiratory gas exchange during exercise. Journal of Applied Physiology, 35: 236-243.

25. McGill R, Tukey JW \& Larsen WA (1978). Variations of Box plots. American Statistician, 32: 12-16.

26. Pratt JW \& Gibbons JD (1981). Concepts of Nonparametric Theory. 2nd edn. Springer-Verlag, New York.

27. Negrão CE, Moreira ED, Santos MC, Farah VM \& Krieger EM (1992). Vagal function impairment after exercise training. Journal of Applied Physiology, 72: 1749-1753.

28. Yamamoto K, Miyachi M, Saitoh T, Yoshioka A \& Onodera S (2001). Effects of endurance training on resting and post-exercise cardiac autonomic control. Medicine and Science in Sports and Exercise, 33: 1496-1502

29. Pichot V, Roche F, Gaspoz JM, Enjolras F, Antoniadis A, Minini P, Costes F, Busso T, Lacour JR \& Barthelemy JC (2000). Relation between heart rate variability and training load in middle-distance runners. Medicine and Science in Sports and Exercise, 32: 17291736 .

30. Migliaro ER, Contreras P, Bech S, Etxagibel A, Castro M, Ricca R \& Vicente K (2001). Relative influence of age, resting heart rate and sedentary life style in short-term analysis of heart rate variability. Brazilian Journal of Medical and Biological Research, 34: 493-500.

31. Malliani A, Pagani M, Lombardi F \& Cerutti S (1991). Cardiovascular neural regulation explored in the frequency domain. Circulation, 84: 482-492.

32. Pagani M, Lombardi F, Guzzetti S, Rimoldi O, Furlan R, Pizzinelli P, Sandrone G, Malfatto G, Dell'Orto S \& Piccaluga E (1986). Power spectral analysis of heart rate and arterial pressure variabilities as a marker of sympatho-vagal interaction in man and conscious dog. Circulation Research, 59: 178-193.

33. Bonaduce D, Petretta M, Cavallaro V, Apicella C, Ianniciello A, Romano M, Breglio R \& Marciano F (1998). Intensive training and 
cardiac autonomic control in high level athletes. Medicine and Science in Sports and Exercise, 30: 691-696.

34. Furlan R, Porta A, Costa F, Tank J, Baker L, Schiavi R, Robertson D, Malliani A \& Mosqueda-Garcia R (2000). Oscillatory patterns in sympathetic neural discharge and cardiovascular variables during orthostatic stimulus. Circulation, 101: 886-892.

35. Hedelin R, Bjerle P \& Henriksson-Larsen K (2001). Heart rate varia- bility in athletes: relationship with central and peripheral performance. Medicine and Science in Sports and Exercise, 33: 13941398.

36. Lee MC (2003). Influence of short-term endurance exercise training on heart rate variability. Medicine and Science in Sports and Exercise, 35: 961-969. 